\title{
Targeted demethylation at the CDKN1C/p57 locus induces human $\beta$ cell replication
}

\author{
Kristy Ou, ${ }^{1}$ Ming Yu, ${ }^{1}$ Nicholas G. Moss, ${ }^{1}$ Yue J. Wang, ${ }^{1}$ Amber W. Wang, ${ }^{1}$ Son C. Nguyen, ${ }^{2}$ Connie Jiang, ${ }^{1}$ Eseye Feleke, ${ }^{3}$ \\ Vasumathi Kameswaran, ${ }^{1}$ Eric F. Joyce, ${ }^{2}$ Ali Naji, ${ }^{1}$ Benjamin Glaser, ${ }^{3}$ Dana Avrahami, ${ }^{3}$ and Klaus H. Kaestner ${ }^{1}$
}

'Department of Genetics and Institute for Diabetes, Obesity and Metabolism, University of Pennsylvania, Philadelphia, Pennsylvania, USA. 2Department of Cenetics, Penn Epigenetics Institute, Perelman School of Medicine, University of Pennsylvania, Philadelphia, Pennsylvania, USA. ${ }^{3}$ Endocrinology and Metabolism Service, Department of Internal Medicine,

Hadassah-Hebrew University Medical Center, Jerusalem, Israel.

\begin{abstract}
The loss of insulin-secreting $\beta$ cells is characteristic among type I and type II diabetes. Stimulating proliferation to expand sources of $\beta$ cells for transplantation remains a challenge because adult $\beta$ cells do not proliferate readily. The cell cycle inhibitor $\mathrm{p} 57$ has been shown to control cell division in human $\beta$ cells. Expression of $\mathrm{p} 57$ is regulated by the DNA methylation status of the imprinting control region 2 (ICR2), which is commonly hypomethylated in Beckwith-Wiedemann syndrome patients who exhibit massive $\beta$ cell proliferation. We hypothesized that targeted demethylation of the ICR2 using a transcription activator-like effector protein fused to the catalytic domain of TET1 (ICR2-TET1) would repress p57 expression and promote cell proliferation. We report here that overexpression of ICR2-TET1 in human fibroblasts reduces p57 expression levels and increases proliferation. Furthermore, human islets overexpressing ICR2-TET1 exhibit repression of p57 with concomitant upregulation of Ki-67 while maintaining glucose-sensing functionality. When transplanted into diabetic, immunodeficient mice, the epigenetically edited islets show increased $\beta$ cell replication compared with control islets. These findings demonstrate that epigenetic editing is a promising tool for inducing $\beta$ cell proliferation, which may one day alleviate the scarcity of transplantable $\beta$ cells for the treatment of diabetes.
\end{abstract}

\section{Introduction}

In 2014, 422 million people worldwide lived with diabetes (1). While patients with type I or severe forms of type II diabetes can manage extreme glucose excursions by administering insulin shortly before a meal, only $\beta$ cell replacement therapy through transplantation of healthy pancreatic islets obtained from carefully screened deceased organ donors provides long-term diabetes reversal without the need for insulin injections $(2,3)$. However, the use of $\beta$ cell replacement therapy is limited by the availability of transplantable donor islets. As a result, there is a concerted effort to produce functionally mature $\beta$ cells from embryonic stem cells and induced pluripotent stem cells, or to identify strategies to enhance $\beta$ cell proliferation $(4,5)$.

Although inducing $\beta$ cell proliferation would appear to be a straightforward approach, it is severely limited by the fact that the proliferative capacity of human $\beta$ cells declines dramatically after early childhood $(6,7)$. This correlates with an age-related accumulation of the senescence effector p16 that induces cell cycle arrest in $\beta$ cells (8). Strikingly, the overgrowth disorder Beckwith-Wiedemann syndrome (BWS) is characterized by an expansion of $\beta$ cell mass - in addition to other tissue types such as the tongue, kid-

\section{Related Commentary: p. 51}

Conflict of interest: The authors have declared that no conflict of interest exists. License: Copyright 2019, American Society for Clinical Investigation.

Submitted: December 11, 2017; Accepted: October 16, 2018

Reference information: J Clin Invest. 2019;129(1):209-214.

https://doi.org/10.1172/JCI99170. neys, liver, and other abdominal organs - that is associated with decreased protein levels of the cell cycle inhibitor p57 (9), a recognized negative regulator of $\beta$ cell replication (10-12).

The cell cycle inhibitor p57 is the protein product of the CDKN1C gene, which is imprinted and regulated by the DNA methylation status of the nearby imprinting control region 2 (ICR2). The ICR2 is a CpG-dense region located on chromosome $11 \mathrm{p} 15.5$ that is hypomethylated on the paternal allele, and hypermethylated on the maternal allele (13). This asymmetrical methylation signature is linked to preferential expression of CDKN1C from the maternal allele through molecular mechanisms that are still not well understood. In the majority of patients with BWS, the ICR2 is hypomethylated on both alleles (12), correlating with deactivation of CDKN1C and an increase in proliferation of $\beta$ cells.

By mimicking the molecular alterations observed in BWS via transcription activator-like effector (TALE) epigenome editing (Figure 1A), we were able to target and demethylate the ICR2 in $\beta$ cells of human islets. We show in this proof-of-principle study that targeted epigenetic editing can be harnessed to induce $\beta$ cell proliferation and model critical aspects of human imprinting disorders.

\section{Results and Discussion}

A TALE-TET1 effector causes specific demethylation of the ICR2 at the CDKN1C locus. TALE proteins are commonly utilized for epigenome editing owing to their customizable yet highly specific DNA-recognition domain and compatibility with numerous chromatin modifiers $(14,15)$. Indeed, a prior study demonstrated the high specificity and limited off-target effects of TALE proteins fused to the catalytic domain of the methylcytosine dioxygenase 

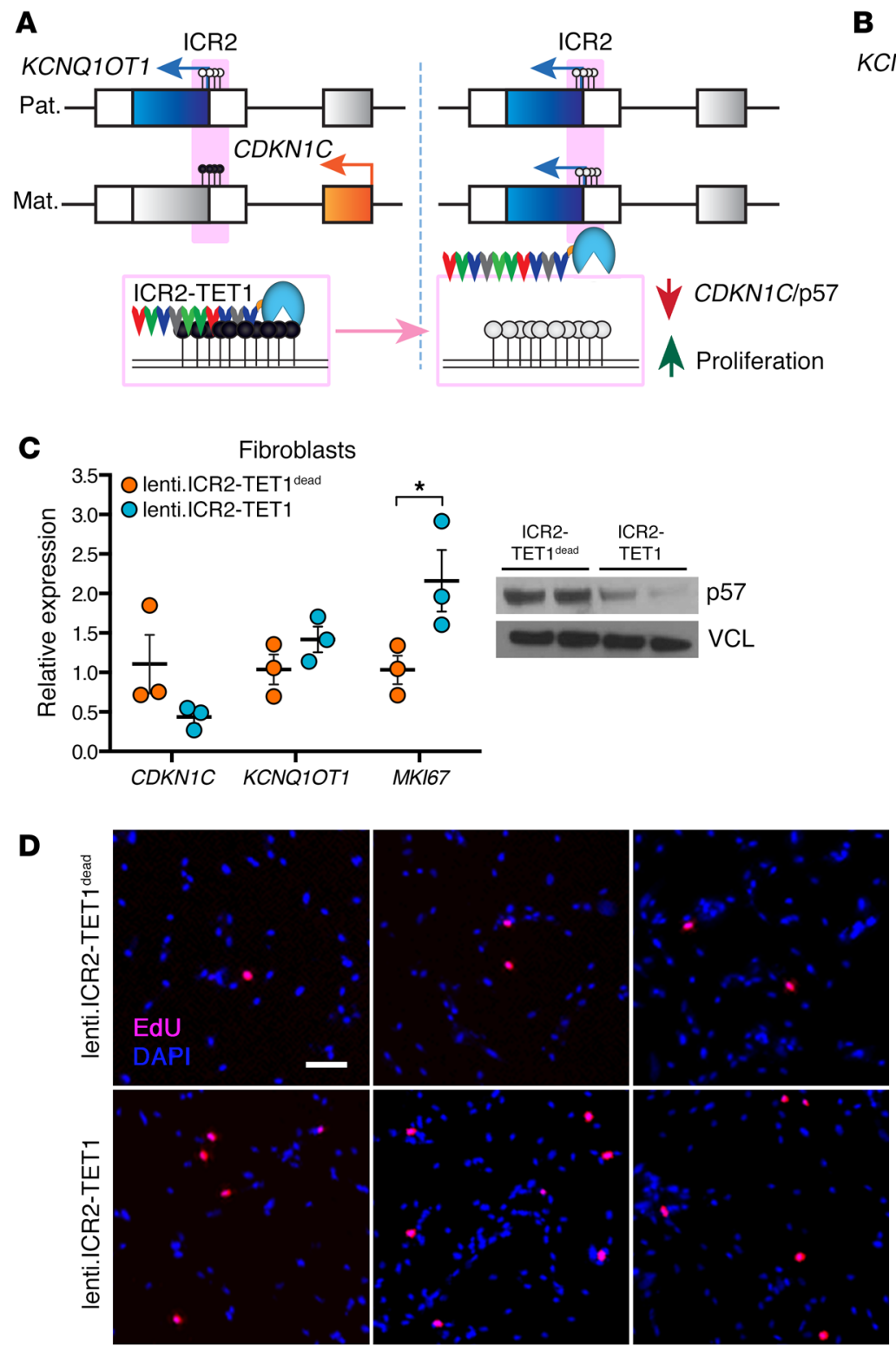

B
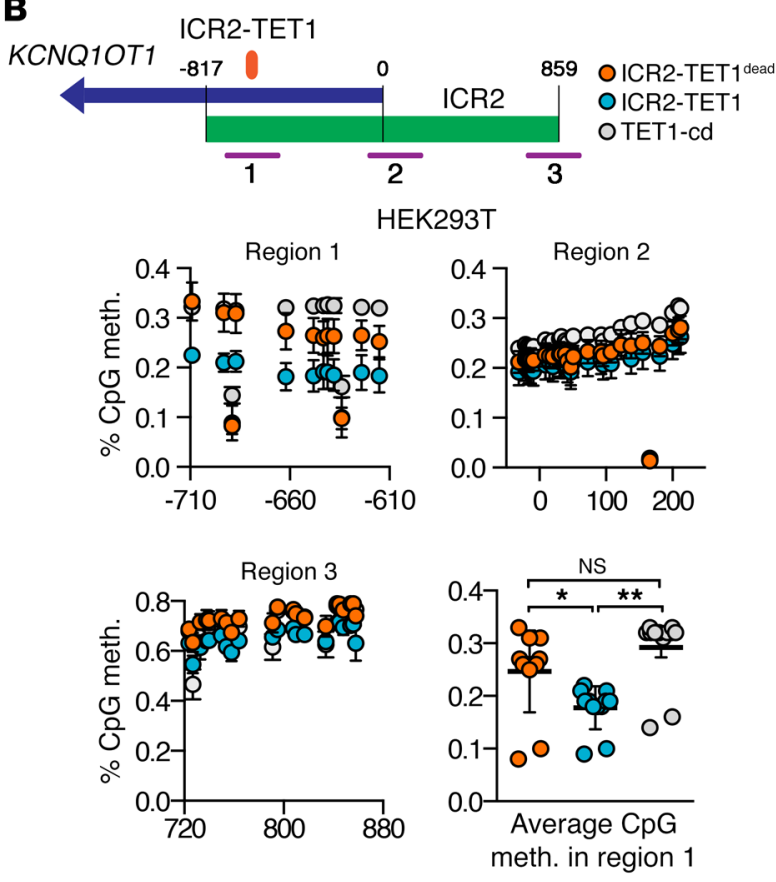

Figure 1. Targeted demethylation of the ICR2 at the CDKN1C/p57 locus causes increased proliferation of human fibroblasts. (A) Schematic of the imprinted chr11p15.5 locus. The ICR2 is methylated (depicted by black circles) at the promoter of the long noncoding RNA KCNO10T1 on the maternal allele, and correlates with maternal allele-specific expression of CDKN1C. A TALE-TET1 fusion protein was designed to target the ICR2 and remove the methylCpGs at the ICR2 in order to deactivate CDKN1C and increase cell proliferation. (B) Three regions within the ICR2 were amplified for methylation analysis by targeted bisulfite sequencing. Percentage $\mathrm{CpG}$ methylation at 3 regions of the ICR2 are shown ( $n=3$ for each condition). (C) p57 mRNA and protein levels in fibroblasts overexpressing ICR2-TET1 dead or ICR2-TET1 ( $n=3$ for each condition). VCL, vinculin. (D) EdU incorporation in fibroblasts 72 hours after transduction with the ICR2-TET1 dead or ICR2-TET1 lentivirus ( $n=5$ for each condition). Scale bar: $100 \mu \mathrm{m}$. ${ }^{*} P<0.05$; ${ }^{* *} P<0.01$ by 1-way ANOVA (B), 1-tailed $t$ test (C), or 2-tailed $t$ test (D). NS, not significant.

TET1 (16), which facilitates the passive and active demethylation of methylated CpGs. We designed a TALE-TET1 fusion protein targeting the ICR2 (ICR2-TET1) at chr11:2,720,607-2,720,625 (Supplemental Figure 1; supplemental material available online with this article; https://doi.org/10.1172/JCI99170DS1). As controls, we engineered a fusion protein with an identical TALE DNA-binding domain ligated to an enzymatically dead TET1 mutant protein (ICR2-TET1 ${ }^{\text {dead }}$ ), and an untethered TET1 catalytic domain (TET1-cd). By performing targeted bisulfite sequencing in sorted HEK293T cells (17), we found that the ICR2-TET1 protein induced demethylation at its binding site in the ICR2 (Figure 1B), demonstrating local specificity of the epimutation achieved. Methylation at regions 2 and 3 of the ICR2, including the KCNQ1OT1 promoter, was not changed by the ICR2-TET1 protein. Furthermore, the untethered TET1-cd had no effect on DNA methylation at the targeted locus. These results establish that the ICR2-targeting TALETET1 protein is functional and a suitable tool for investigating the relationship between the ICR2 methylation status, p57 expression, and proliferative capacity of epigenetically edited cells. It is important to note that the demethylation effect of the TALE-TET1 
A

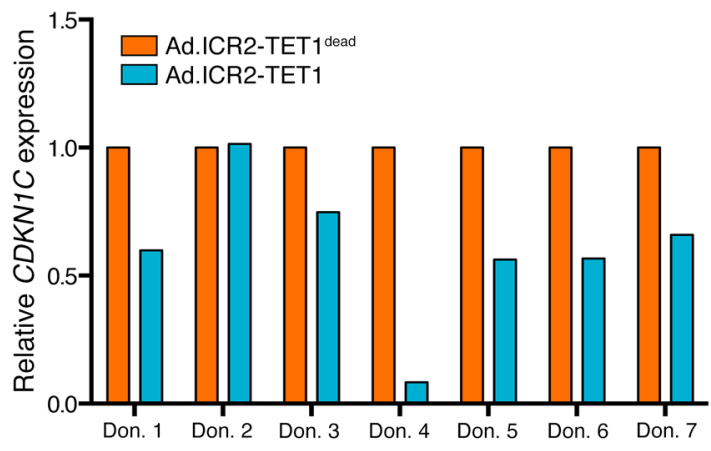

B

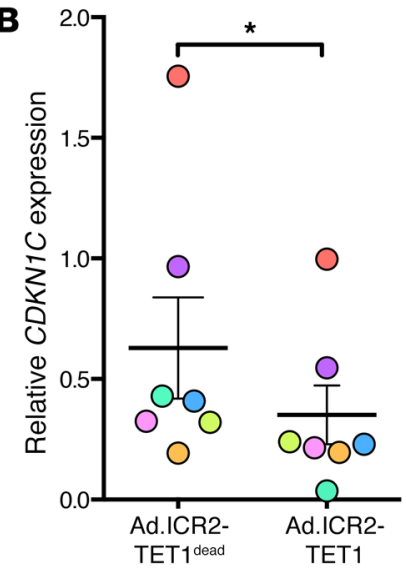

C

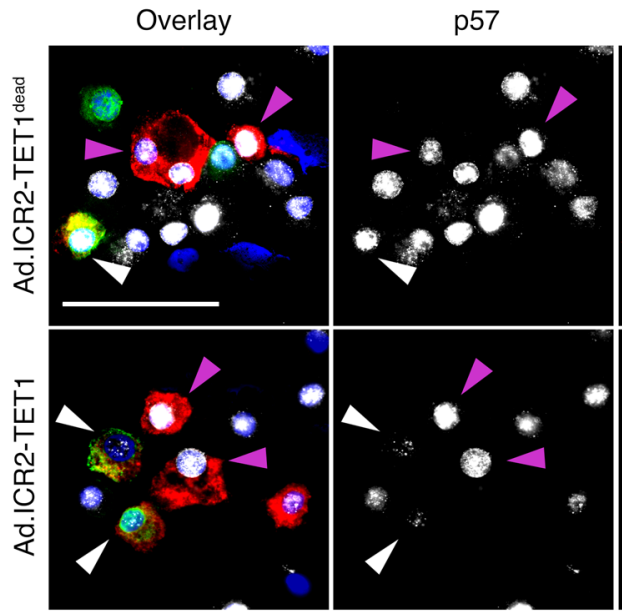

C-peptide

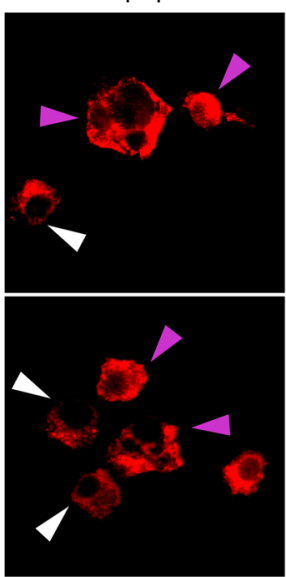

GFP

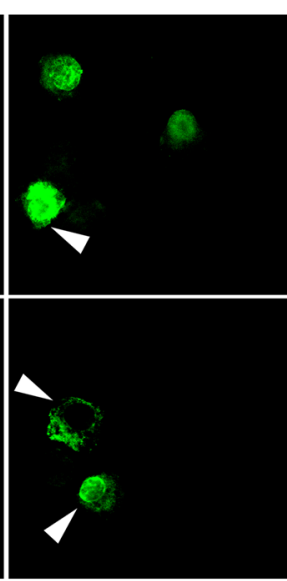

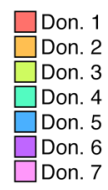

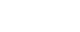

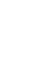

(1)

Figure 2. ICR2-TET1 deactivates $\mathbf{p 5 7}$ in human $\boldsymbol{\beta}$ cells. (A) CDKN1C mRNA expression in islets transduced for 72 hours with the ICR2-TET1 dead or ICR2TET1 adenovirus. Islets were obtained from 7 nondiabetic, nonobese, deceased organ donors. (B) CDKN1C mRNA expression in islets grouped by adenoviral treatment. Each data point represents the relative expression of $C D K N 1 C$ from one of the donors from $\mathbf{A}\left(n=7\right.$ for each condition). ${ }^{*} P<0.02$ by Wilcoxon's matched-pairs signed-rank test. (C) Immunocytochemical staining of transduced $\beta$ cells. White arrowheads indicate transduced GFP ${ }^{+}$ $\beta$ cells, while purple arrowheads indicate untransduced controls. Unlike the Ad.ICR2-TET1 ${ }^{\text {dead }}$-transduced $\beta$ cells, Ad.ICR2-TET1-transduced $\beta$ cells stained weakly for p57. Scale bar: $50 \mu \mathrm{m}$.

system is likely underestimated by bisulfite sequencing due to the protection of hydroxymethylcytosines (the first oxidation product of methylcytosine catalyzed by TET1) from deamination by the sodium bisulfite treatment.

Targeted demethylation of the ICR 2 decreases expression of $p 57$ in cell lines. Next, we assessed whether or not targeted demethylation of the ICR2 reduces the expression of the cell cycle inhibitor CDKN1C. In sorted HEK293T cells, there was a significant 10-fold reduction in CDKN1C expression levels in the ICR2TET1 group compared with the ICR2-TET1 ${ }^{\text {dead }}$ control (Supplemental Figure 2), confirming that gene silencing resulted from TALE-TET1-induced demethylation and not through simple steric hindrance by binding of the TALE fusion protein itself. Human fibroblasts transduced with a lentivirus overexpressing ICR2-TET1 also exhibited a decrease in p57 mRNA and protein levels when compared with ICR2-TET1 $1^{\text {dead }}$ controls (Figure 1C). In addition to downregulation of $\mathrm{p} 57$, we also detected activation of MKI67 encoding Ki-67, a marker of proliferation, in transduced human fibroblasts (Figure 1C). These results demonstrate a causal relationship between the DNA methylation status of the ICR2 and CDKN1C gene expression.

The mechanism by which the methylation status of the ICR2 regulates $C D K N 1 C$ expression is unclear. The long noncoding RNA KCNQ1OT1, which is transcriptionally active when the ICR2 is hypomethylated, has been suggested to silence CDKN1C in cis $(18,19)$. Although we observed a slight increase in expression levels of KCNQ1OT1 in both transfected HEK293T cells and transduced fibroblasts (Figure 1C and Supplemental Figure 2), we did not find any significant increase in bi-allelic expression of KCNQ1OT1 in response to ICR2 demethylation by DNA-RNA FISH (Supplemental Figure 3), indicating that deactivation of CDKN1C is mediated by a mechanism independent of the in cis function of KCNQ1OT1 $(20,21)$.

Demethylation of the ICR2 increases proliferation in human fibroblasts. Having established that targeted demethylation of the ICR2 causes inactivation of p57 and increased expression of Ki-67 in human fibroblasts, we wanted to evaluate the phenotypic outcome of TALE-TET1-induced demethylation of the ICR2. We performed 

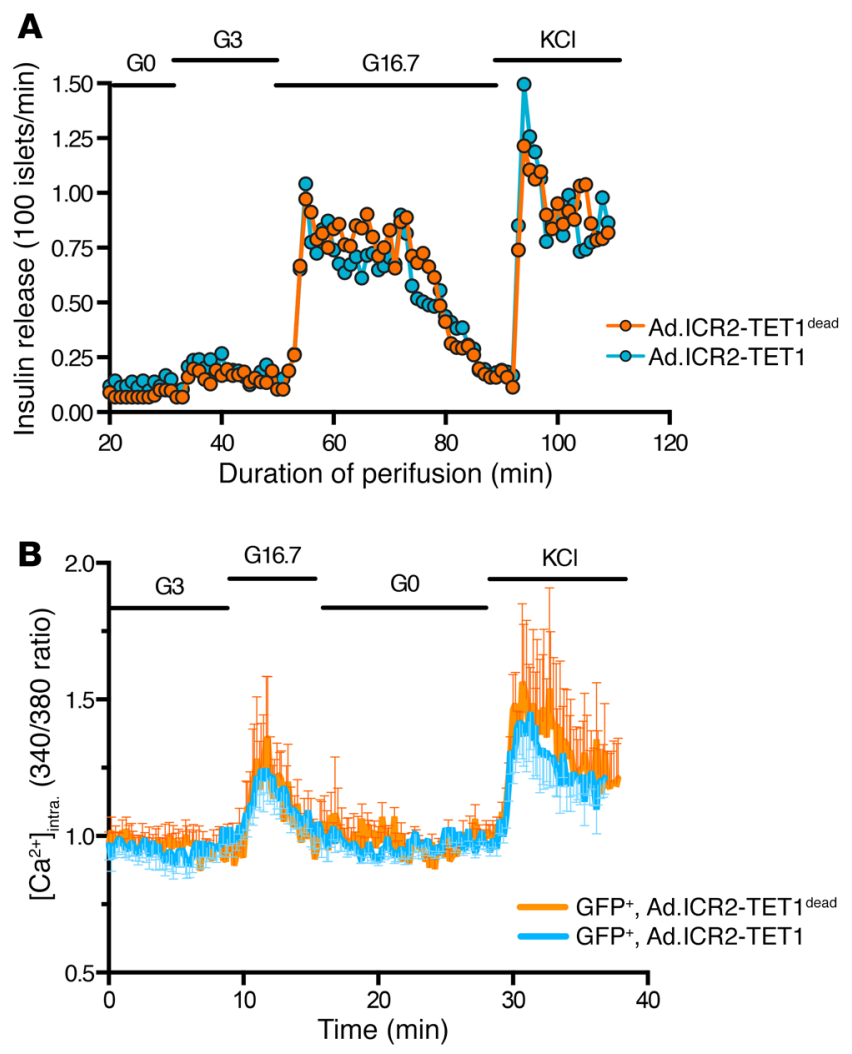

Figure 3. Epigenetically edited $\beta$ cells retain glucose-sensing behavior. (A) Glucose-stimulated insulin secretion in Ad.ICR2-TET1 dead - and Ad.ICR2TET1-transduced islets. (B) $\left[\mathrm{Ca}^{2+}\right]_{\mathrm{i}}$ levels in Ad.ICR2-TET1 dead $\mathrm{GFP}^{+}(n=5)$ and Ad.ICR2-TET1 GFP ${ }^{+}(n=3) \beta$ cells in response to 0,3 , and $16.7 \mathrm{mM}$ glucose (G0, $\mathrm{G3}$, and $\mathrm{C} 16.7)$, and $30 \mathrm{mM} \mathrm{KCl}$ stimulation. Values on the $y$ axis are the $340 / 380 \mathrm{~nm}$ excitation ratio of the calcium indicator Fura-2AM.

an in vitro proliferation assay by quantifying incorporation of the thymidine analog 5-ethynyl-2'-deoxyuridine (EdU) into actively synthesizing DNA strands of human fibroblasts that have been transduced by the ICR2-TET1 or ICR2-TET1 ${ }^{\text {dead }}$ control lentivirus. The percentage of EdU-positive cells in the ICR2-TET1-treated fibroblasts was significantly increased compared with ICR2TET1 ${ }^{\text {dead }}$ controls (Figure 1D), confirming that our strategy to generate an epimutation at the ICR2 can indeed induce cell proliferation.

p57 levels are reduced in epigenetically edited human pancreatic $\beta$ cells. Pursuing our ultimate goal of epigenetic editing by ICR2TET1 towards increasing $\beta$ cell mass, we employed our TALE-TET1 strategy in human islets obtained from nondiabetic, nonobese, deceased organ donors. Because of low efficiency of transduction with lentiviral vectors (data not shown), we transferred our ICR2-TET1 and ICR2-TET1 ${ }^{\text {dead }}$ constructs into adenoviral vectors. Consistent with our observations in human fibroblasts, islets transduced with an adenovirus overexpressing ICR2-TET1 displayed decreased expression levels of CDKN1C (Figure 2A). Considering that the adenovirus transduced only a subset of the islet cells (Supplemental Figure 4), the actual decrease in CDKN1C expression in the transduced $\beta$ cells is likely underestimated. Importantly, several independent transcriptomic studies had reported that CDKN1C is expressed primarily in $\beta$ cells compared with other islet endocrine cell types (22-24), suggesting that the decrease in CDKN1C expression resulting from ICR2-TET1 overexpression primarily occurred in $\beta$ cells. Donor-to-donor variability in CDKN1C deactivation can be attributed to variability in transduction efficiency, where on average only $20 \%-40 \%$ of $\beta$ cells were successfully transduced. Despite variation between the individual donors assayed, there was a significant decrease in $C D K N 1 C$ transcript levels in the ICR2-TET1-treated islets when all donors were analyzed collectively $(P<0.05)$ (Figure 2B). Decreased p 57 protein levels were further verified through immunocytochemical staining of transduced compared with nontransduced $\beta$ cells (Figure 2C).

Epigenetically edited $\beta$ cells retain glucose-sensing functionality. Next, we wanted to determine whether p57 deactivation impacted $\beta$ cell function. We first performed whole islet perifusion to determine differences in glucose-stimulated insulin secretion (GSIS) between Ad.ICR2-TET1 ${ }^{\text {dead }}$ - and Ad.ICR2-TET1-transduced islets. We found that GSIS was not impaired in Ad.ICR2-TET1-transduced islets compared with Ad.ICR2-TET1 $1^{\text {dead }}$ controls (Figure 3A). However, only a subset of $\beta$ cells were transduced in the whole islet, necessitating the need for an additional technique to assess functionality of the transduced $\beta$ cells specifically. We measured single-cell intracellular calcium concentrations as a surrogate for insulin secretion in transduced $\beta$ cells by using Fura- 2 as a calcium indicator. Basal $\left[\mathrm{Ca}^{2+}\right]_{\mathrm{i}}$ as well as calcium response to high-glucose stimulation were similar between $\mathrm{GFP}^{+}$, Ad.ICR2-TET1-transduced $\beta$ cells and GFP $^{+}$, Ad.ICR2-TET1 ${ }^{\text {dead }}$ control cells (Figure 3B). Together, these results demonstrate that targeted demethylation of the ICR2 does not negatively impact $\beta$ cell function.

Targeted demethylation of the ICR2 leads to increased replication of $\beta$ cells. After verifying that epigenetically edited $\beta$ cells displayed normal glucose-sensing behavior, we next assessed the effect of targeted ICR2 demethylation on $\beta$ cell replication. In addition to decreased CDKN1C transcript levels, ICR2-TET1-treated human islets exhibited increased expression of the proliferation marker MKI67, which was significantly anticorrelated with $C D K N 1 C$ transcript levels (Figure 4A). We were also able to detect Ki-67 protein in transduced $\beta$ cells, as well as quantify an increase in expression of several cell cycle genes controlling the G2/M transition (Supplemental Figure 5).

In order to study the long-term effect of TALE-TET1-induced epimutation on $\beta$ cell proliferation, we utilized the immunodeficient NOD-scid IL2ry ${ }^{\text {null }}$ (NSG) mouse model rendered diabetic with treatment of streptozotocin (Figure 4B). To control for the variability of mitogenic stimuli provided by the host, each diabetic NSG mouse was transplanted simultaneously with 100 Ad.ICR2-TET1-

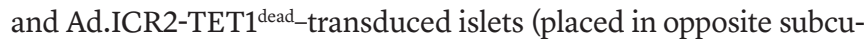
taneous pockets). After transplantation, mice were administered BrdU in the drinking water for 3 weeks in order to label every cell that entered S-phase during this time frame. The transplanted islets were able to correct the hyperglycemic glucose levels of the NSG mice, indicating that they remain functional (Supplemental Figure 6). After 3 weeks, the islet xenografts were harvested, processed for paraffin sectioning, and stained for C-peptide and BrdU to identify $\beta$ cells and cells that replicated their DNA, respectively. Strikingly, we observed an increase in the percentage of BrdU-positive $\beta$ cells in the islet fraction that had received the ICR2-TET1 compared with the ICR2-TET1 ${ }^{\text {dead }}$ control in 4 out of the 5 donors that underwent the transplant study (Figure 4, C and D). Furthermore, we detect- 

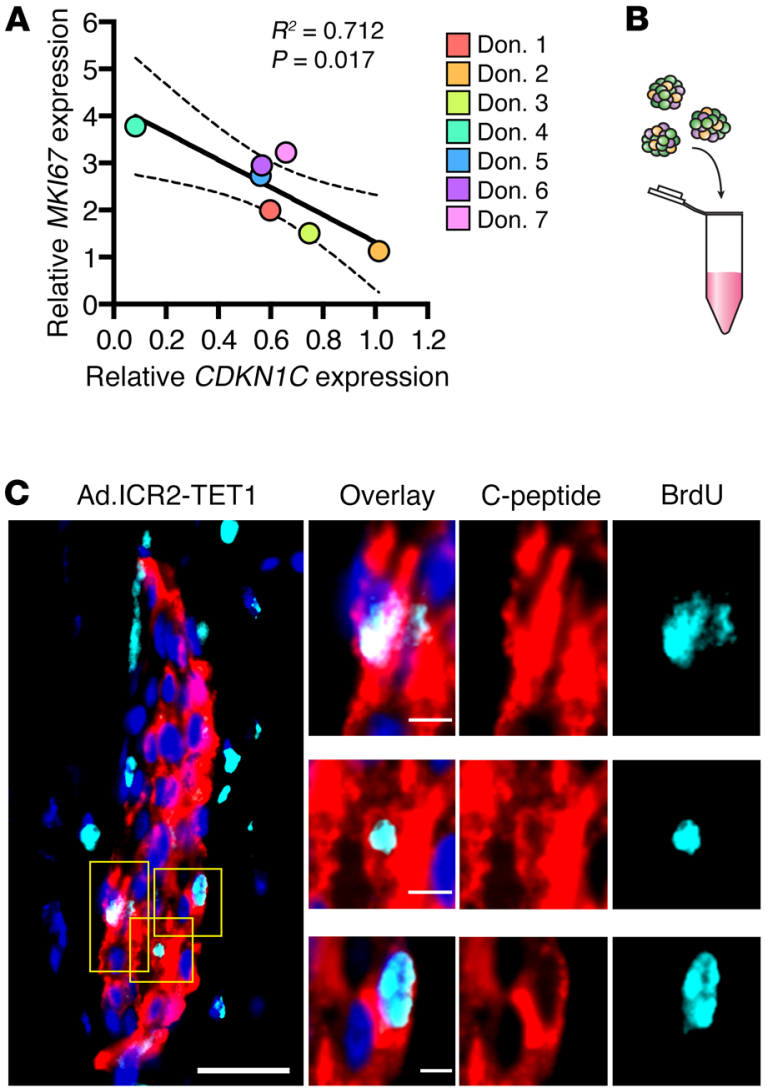

B
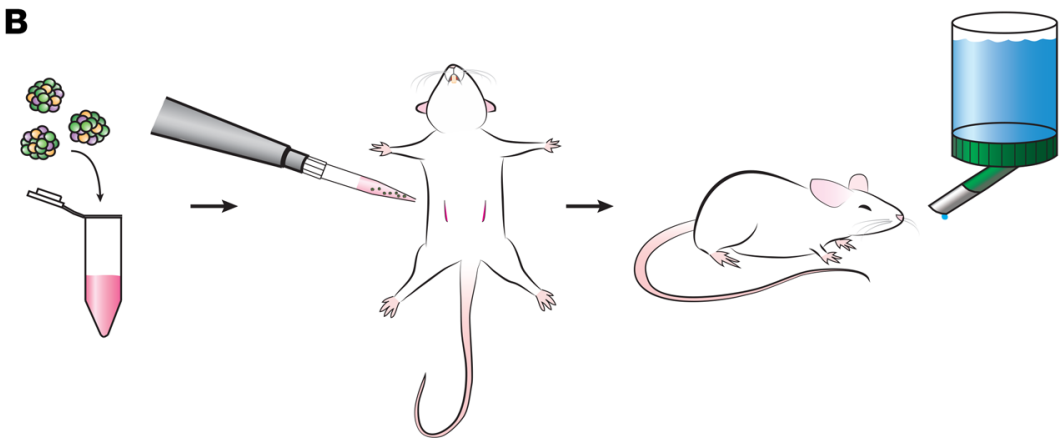

$\mathbf{E}$

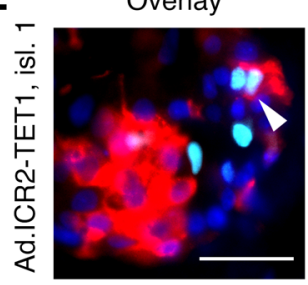

C-peptide

BrdU
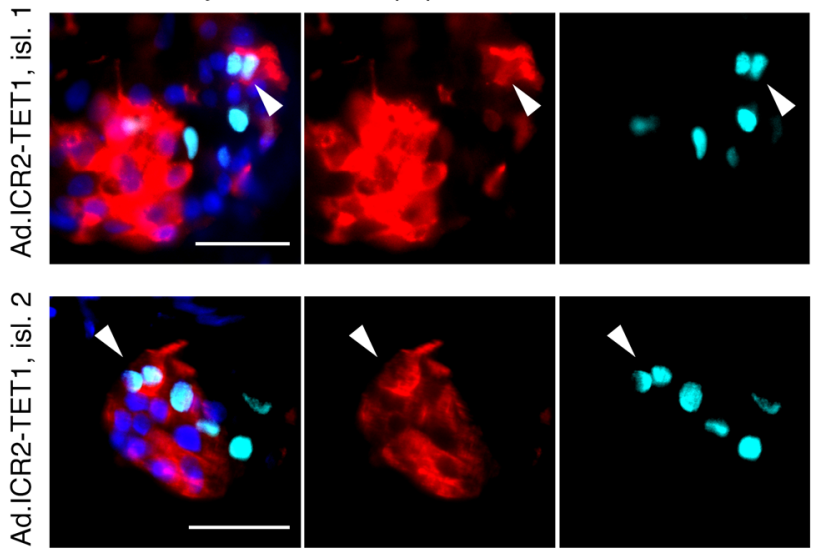

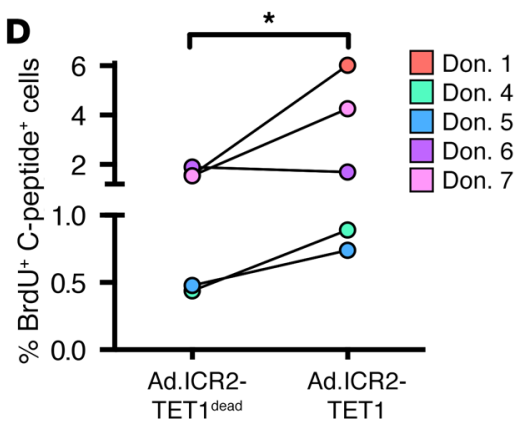

Figure 4. Targeted epimutation at the ICR2 induces human $\beta$ cell replication. (A) Expression of CDKN1C mRNA is significantly anticorrelated with mRNA expression levels of the proliferation marker MKI67 in islets transduced with Ad.ICR2-TET1. mRNA levels were normalized to HPRT1. Each data point represents a single donor. (B) Schematic of subcutaneous transplantation of transduced human islets in diabetic NSG mice. For each donor, 100 islets transduced with the ICR2-TET1 ${ }^{\text {dead }}$ or ICR2-TET1 adenovirus were transplanted in the left or right subcutaneous abdominal incision, respectively. Mice were administered BrdU via the drinking water for 3 weeks before the grafts were harvested. (C) Example of an islet transduced with Ad.ICR2-TET1 with $3 \mathrm{BrdU}+\beta$ cells. For each donor and treatment, 800 to 2,000 C-peptide ${ }^{+}$cells were counted. Scale bars: $50 \mu \mathrm{m}$ and $10 \mu \mathrm{m}$ (insets). (D) Percentage of $\mathrm{BrdU}^{+} \beta$ cells in sectioned xenografts 3 weeks after transplant. Each pair of data points represents an individual donor. ${ }^{*} P<0.05$ by ratio paired $t$ test. (E) BrdU/C-peptide double-positive doublets, indicated by the white arrowheads, in Ad.ICR2-TET1-transduced xenografts. Scale bars: $50 \mu \mathrm{m}$. ed BrdU-positive doublets, which suggests that the transduced $\beta$ cells are capable of completing mitosis (Figure $4 \mathrm{E}$ ). These results demonstrate the applicability of epigenetic editing for promoting $\beta$ cell replication. It is notable that targeted demethylation of the ICR2 also led to an increase in proliferation of $\alpha$ cells, which express $C D K N 1 C$ at lower levels than $\beta$ cells (Supplemental Figure 7). Thus, the development of an adenoviral or adeno-associated viral system specifically targeting $\beta$ cells will be necessary to move epimutation of the ICR2 using TALE-TET1 closer to therapeutic applications.

We demonstrate that epigenetic editing can be applied to promote replication in human pancreatic $\beta$ cells. We successfully demonstrate that targeted demethylation of the ICR2 directly results in the downregulation of p57, aligning with the underlying epigenetic cause of BWS. In $\beta$ cells, this epimutation causes increased Ki-67 expression as well as increased cell cycle entry as detected by BrdU incorporation in the xenografts of transplanted NSG mice.

Epigenetic editing holds great therapeutic potential due to its effectiveness at reprogramming stable epigenetic marks to modu- late expression of disease-related genes. Already, several studies have demonstrated the promise of epigenetic editing in targeting genes involved in cancer and neurological disorders (25-27). In the context of stimulating $\beta$ cell replication, targeting $\mathrm{p} 57$ through epigenetic editing is an attractive strategy for several reasons. First, p57 is difficult to inhibit pharmacologically due to its nuclear localization, necessitating intervention at the gene regulation level. Secondly, the DNA methylation signature of the epigenetically edited parent cell is inherited by the daughter cells after cell division, which suggests that CDKN1C will remain silenced after multiple rounds of cell division. However, additional studies are needed to investigate how long the induced epimutation and p57 deactivation can be maintained, and to evaluate the risk for hyperinsulinism from continuous $\beta$ cell expansion. Our data suggest that epigenetically edited $\beta$ cells have functional $\mathrm{K}_{\text {ATP }}$ channels, and thus should be responsive to diazoxide to attenuate insulin secretion if needed. Altogether, our results for using epigenetic editing to promote $\beta$ cell replication is an encouraging 
starting point towards increasing the supply of transplantable $\beta$ cells for diabetic patients.

\section{Methods}

For complete methods, see supplemental material.

Statistics. Student's $t$ test using a 2-tailed distribution, unless otherwise stated in the text or figure legends, was used to determine statistical significance. For all tests, $P<0.05$ was considered statistically significant. Data represent mean \pm SEM. All analyses were conducted using GraphPad Prism 6.

Study approval. The experiments using islets obtained from cadaveric organ donors were declared exempt by the IRB of the University of Pennsylvania. The transplantation studies were approved by the IACUC of the University of Pennsylvania.

\section{Author contributions}

KHK, BG, and DA conceived the study. KO, MY, KHK, BG, and DA designed the experiments. KO, MY, CJ, YJW, and NGM acquired the data. KO and YJW analyzed and interpreted the data. KO wrote the manuscript and performed the statistical analyses. AWW, SCN, EFJ, VK, EF, and AN provided material support. KHK, BG,
YJW, and EFJ reviewed and edited the manuscript. KHK supervised the study.

\section{Acknowledgments}

We would like to thank the Kaestner lab for helpful suggestions and discussions. This work was funded by the National Heart, Lung, and Blood Institute (NHLBI) grant 2T32HL007954-16 (to KO), and the National Institute of Diabetes and Digestive and Kidney Diseases (NIDDK) grants U01-DK089529 and R01-DK088383 (to KHK). This research was performed using resources and/or funding provided by the NIDDK-supported Human Islet Research Network (HIRN, RRID:SCR_014393; https://hirnetwork.org; UC4 DK104119) to KHK, DA, and BG. We acknowledge support of the Islet Cell Biology Core and Functional Genomics Core of Penn's Diabetes Research Center (P30-DK019525).

Address correspondence to: Klaus H. Kaestner, Department of Genetics, University of Pennsylvania, 12-126 Smilow Center of Translational Research, 3400 Civic Center Blvd, Philadelphia, Pennsylvania 19104, USA. Phone: 215.898.8759; Email: kaestner @pennmedicine.upenn.edu.
1. Centers for Disease Control and Prevention. National Diabetes Statistics Report, 2017. Atlanta, Georgia, USA: Centers for Disease Control and Prevention, US Department of Health and Human Services; 2017. https://www.cdc.gov/ diabetes/pdfs/data/statistics/nationaldiabetes-statistics-report.pdf.

2. Rickels MR, et al. Long-term improvement in glucose control and counterregulation by islet transplantatxeeion for type 1 diabetes. JClin Endocrinol Metab. 2016;101(11):4421-4430.

3. Hering BJ, et al. Phase 3 trial of transplantation of human islets in type 1 diabetes complicated by severe hypoglycemia. Diabetes Care. 2016;39(7):1230-1240.

4. Wang P, Fiaschi-Taesch NM, Vasavada RC, Scott DK, García-Ocaña A, Stewart AF. Diabetes mellitus - advances and challenges in human $\beta$-cell proliferation. Nat Rev Endocrinol. 2015;11(4):201-212.

5. Millman JR, Xie C, Van Dervort A, Gürtler M, Pagliuca FW, Melton DA. Generation of stem cell-derived $\beta$-cells from patients with type 1 diabetes. Nat Commun. 2016;7:11463.

6. Wang YJ, et al. Single-cell mass cytometry analysis of the human endocrine pancreas. Cell Metab. 2016;24(4):616-626.

7. Maedler K, et al. Aging correlates with decreased beta-cell proliferative capacity and enhanced sensitivity to apoptosis: a potential role for Fas and pancreatic duodenal homeobox-1. Diabetes. 2006;55(9):2455-2462.

8. Köhler CU, Olewinski M, Tannapfel A, Schmidt WE, Fritsch H, Meier JJ. Cell cycle control of $\beta$-cell replication in the prenatal and postnatal human pancreas. Am J Physiol Endocrinol Metab.
2011;300(1):E221-E230.

9. Kalish JM, et al. Congenital hyperinsulinism in children with paternal 11p uniparental isodisomy and Beckwith-Wiedemann syndrome. JMed Genet. 2016;53(1):53-61.

10. Avrahami D, et al. Targeting the cell cycle inhibitor $\mathrm{p} 57 \mathrm{Kip} 2$ promotes adult human $\beta$ cell replication. J Clin Invest. 2014;124(2):670-674.

11. Ueberberg S, et al. Differential expression of cell-cycle regulators in human beta-cells derived from insulinoma tissue. Metab Clin Exp. 2016;65(5):736-746.

12. Calvello M, et al. Quantitative DNA methylation analysis improves epigenotype-phenotype correlations in Beckwith-Wiedemann syndrome. Epigenetics. 2013;8(10):1053-1060.

13. Diaz-Meyer N, et al. Silencing of CDKN1C (p57KIP2) is associated with hypomethylation at KvDMR1 in Beckwith-Wiedemann syndrome. JMed Genet. 2003;40(11):797-801.

14. Amabile A, et al. Inheritable silencing of endogenous genes by hit-and-run targeted epigenetic editing. Cell. 2016;167(1):219-232.e14.

15. Mussolino C, et al. TALENs facilitate targeted genome editing in human cells with high specificity and low cytotoxicity. Nucleic Acids Res. 2014;42(10):6762-6773.

16. Maeder ML, et al. Targeted DNA demethylation and activation of endogenous genes using programmable TALE-TET1 fusion proteins. Nat Biotechnol. 2013;31(12):1137-1142.

17. Bernstein DL, Le Lay JE, Ruano EG, Kaestner KH. TALE-mediated epigenetic suppression of CDKN2A increases replication in human fibroblasts. J Clin Invest. 2015;125(5):1998-2006.
18. Mancini-Dinardo D, Steele SJ, Levorse JM, Ingram RS, Tilghman SM. Elongation of the Kcnq1ot1 transcript is required for genomic imprinting of neighboring genes. Genes Dev. 2006;20(10):1268-1282.

19. Green K, et al. A developmental window of opportunity for imprinted gene silencing mediated by DNA methylation and the Kcnq1ot1 noncoding RNA. Mamm Genome. 2007;18(1):32-42.

20. Fitzpatrick GV, et al. Allele-specific binding of CTCF to the multipartite imprinting control region KvDMR1. Mol Cell Biol. 2007;27(7):2636-2647.

21. Kanduri C, et al. A differentially methylated imprinting control region within the Kenq1 locus harbors a methylation-sensitive chromatin insulator. J Biol Chem. 2002;277(20):18106-18110.

22. Muraro MJ, et al. A single-cell transcriptome atlas of the human pancreas. Cell Syst. 2016;3(4):385-394.e3.

23. Bramswig NC, et al. Epigenomic plasticity enables human pancreatic $\alpha$ to $\beta$ cell reprogramming. J Clin Invest. 2013;123(3):1275-1284.

24. Wang YJ, et al. Single-cell transcriptomics of the human endocrine pancreas. Diabetes. 2016;65(10):3028-3038.

25. Falahi F, et al. Towards sustained silencing of HER2/neu in cancer by epigenetic editing. $\mathrm{Mol}$ Cancer Res. 2013;11(9):1029-1039.

26. Heller EA, et al. Locus-specific epigenetic remodeling controls addiction- and depression-related behaviors. Nat Neurosci. 2014;17(12):1720-1727.

27. Garcia-Bloj B, et al. Waking up dormant tumor suppressor genes with zinc fingers, TALEs and the CRISPR/dCas9 system. Oncotarget. 2016;7(37):60535-60554. 